\title{
Knowledge and Coping Strategies Among Patients Diagnosed With Type 2 Diabetes Mellitus
}

\author{
Maram A. Najjar ${ }^{1}$, Waddah M. D'emeh ${ }^{2} \&$ Mohammed Ibrahim Yacoub $^{2}$ \\ ${ }^{1}$ College of Nursing \& Midwifery, Hebron University, Hebron, West Bank \\ ${ }^{2}$ School of Nursing, The University of Jordan, Amman, Jordan \\ Correspondence: Waddah M. D'emeh, School of Nursing, The University of Jordan, Queen Rania Street, Amman \\ 11942, Jordan. Tel: 962-5355-000, ext. 23142. E-mail: w.demeh@ju.edu.jo
}

Received: November 19, 2019 Accepted: Janurary 22, 2020 Online Published: Janurary 30, 2020

doi:10.5539/gjhs.v12n2p69

URL: https://doi.org/10.5539/gjhs.v12n2p69

\begin{abstract}
The purposes of this study were to investigate level of knowledge and employed coping strategies, and to examine the relationship between knowledge and coping strategies among persons diagnosed with Type 2 diabetes. Cross sectional descriptive design was used. A convenience sample of 222 adult patients with diabetes was recruited. Diabetes knowledge test (DKT) and the Diabetes Coping Measure (DCM) were used. The overall knowledge test score was low. Participants achieved higher scores in tackling spirit and diabetes integration coping while avoidance coping strategies had the lowest scores. There was a significant positive correlation between knowledge and tackling spirit coping and diabetes integration, and a significant negative correlation between knowledge and passive resignation coping. Knowledge among patients with type 2 diabetes was poor. Several areas of knowledge deficits were identified. Efforts to improve knowledge of persons with diabetes need to be continued along with an emphasis on their coping strategies used which require assessment and understanding by health care providers in clinical settings.
\end{abstract}

Keywords: type 2 diabetes, diabetes knowledge, coping strategies, diabetes knowledge test, diabetes coping measure

\section{Introduction}

Diabetes is considered a major public health problem (Unnikrishnan \& Mohan, 2016). It is a disabling disease associated with significant morbidity and mortality (Zhu et al., 2015). Individuals with diabetes who have a high level of knowledge regarding diabetes self-care, tend to have a better long term glycemic control (Islam et al., 2015; Gomes et al., 2018) and good self-care practices (Kassahun, Gesesew, Mwanri, \& Eshetie, 2016). Lack of knowledge is one of the barriers of self-management and medication adherence among this population (Ahola \& Groop, 2013). In addition, persons with diabetes need to deal with daily stressful challenges of their illness. Unfortunately, diabetes could lead them to distress and eventually to depression (Mulligan et al., 2017; Ridosh, Roux, Meehan, \& Penckofer, 2017).

Jordan has a diabetes prevalence rate of $11.7 \%$ with an additional $7.2 \%$ of impaired glucose tolerance (International Diabetes Federation [IDF], 2015) . This high prevalence rate of diabetes and the anticipated increase in the number of persons with diabetes in Jordan over the next two decades are considered an alarming signs which requires a careful assessment of knowledge status among those who have been diagnosed with the disease. Baseline assessment of Jordanian patients' knowledge about diabetes could help in designing future educational programs aim to overcome the burden of diabetes and its complication.

\subsection{Aims}

The purpose of this study was to assess the level of diabetes-related knowledge and the employed coping strategies among persons diagnosed with type 2 diabetes in Jordan. Specifically, current study answered the following research questions: (1) What is the level of knowledge among persons diagnosed with type 2 diabetes? (2) What are the coping strategies employed by persons diagnosed with type 2 diabetes? (3) Is there a relationship between the level of knowledge of diabetes among persons diagnosed with type 2 diabetes and their employment of coping strategies? 


\section{Methods}

\subsection{Design}

A cross sectional descriptive-correlational design was used to assess knowledge and coping strategies among people diagnosed with type 2 diabetes.

\subsection{Setting}

Participants were recruited from 3 hospitals and 4 outpatient's clinics located in the center district of Jordan. According to the Jordanian Ministry of Health, around $72 \%$ of all hospital beds are located in the center district of Jordan (Ministry of Healh [MOH], 2015). Data were collected over 4 months (Jan. to April 2017).

\subsection{Sample}

A non-probability, convenience sampling technique was used to recruit study participants. The inclusion criteria included: (i) a patient who had diagnosed with type 2 diabetes and (ii) are able to read and speak Arabic language. The minimum required sample size was estimated using $\mathrm{G}^{*}$ Power software version 3.10 (Faul, Erdfelder, Lang \& Buchner, 2007). Using Analysis of variance (ANOVA) test with a moderate effect size of 0.25 , and a power of 0.80 , and 0.05 level of significant, the estimated sample size was 200 patients. A total of 222 patients diagnosed with type 2 diabetes were composed the final sample for this study.

\subsection{Instrumentation}

In this study, two self-administered questionnaires were used with permission. The first instrument was the revised Brief Diabetes Knowledge Test (DKT) (Fitzgerald et al., 2016). The (DKT) is composed of 23 multiple choice questions divided in two sections. The first section has 14 items, assesses the general knowledge. The second section constitute of 9 items for insulin use. The DKT is a reliable and valid tool for the assessment of knowledge among diabetic patients. For the purpose of this study, the first section with 14 items was used since it is specific for Type 2 diabetes. It was translated to the Arabic language following the World Health Organization (WHO) guideline for translation ("World Health Organization," n.d.).

The second instrument was The Diabetes Coping Measure (DCM) scale. It was used to determine behavioral and cognitive coping. The DCM is composed of 21 items and has four subscales: tackling spirit (5 items), passive resignation (5 items), avoidance (5 items), and diabetes integration (6 items) (Welch, 1994). The items are scored using 5-point likert scale: disagree strongly, disagree a little, don't know, agree a little and agree strongly. Subscales are scored from 0 to 100, with higher scores reflecting better use of coping strategies (Welch, Jacobson \& Polonsky, 1997).

\subsection{Ethical Consideration}

Before starting data collection, the ethical approval was obtained from the ethical and the Institutional Review board from each setting. Participants were informed regarding the privacy and confidentiality of the study, specifically, participants were informed that all information will be used for research purpose only and no one other than the researchers can access it, the questionnaires did not include names, or personal information.

\subsection{Data Collection Procedure}

Prior to data collection, ethical approval was obtained from the ethical committee of the participating institutions. Data were collected through self-administering questionnaire. The researcher visited the targeted institutions; met the head of each department/ clinics or the person who was in charge to inform him/her about the study, also to determine the available units and outpatient clinics that were targeted for data collection. Those who expressed interest in participation were approached by the researcher who explained the purpose of the study, its significance, and assuring the patients that the study was anonymous and that they have the right to accept or refuse participation in the study without any direct or indirect harm. A package of a cover letter, consent form, two self-report questionnaires and an author-developed demographic survey was distributed to all patients who expressed interest in participation in the study. All questionnaires were kept in a cabinet in researcher's office and all electronic data were kept in the principal researcher's personal computer.

\subsection{Data Analysis}

Data were analyzed using the Statistical Package for the Social Science (SPSS) version 21. Descriptive statistics including mean and Standard Deviation (SD) for continuous variables and frequencies for categorical variables was conducted. Correlation test was used to assess the relationship between knowledge and coping strategies, it was also used to assess the relationship between knowledge and sociodemographic variables and between coping strategies and sociodemographic variables. Data was considered statistically significant at $\alpha<.05$. 


\section{Results}

\subsection{Patient's Demographic Characteristics}

A total of 222 persons with diabetes were recruited for this study. Approximately half of the sample was from governmental sector and the majority of participants were recruited from outpatient clinics. More than half of study sample were currently unemployed, had a family history of diabetes and almost half of them had other co-morbidities, while fewer had no diabetes complications. Participants had an average of 9 years for having a diabetes diagnosis (Table 1).

Table 1. Patients Demographic Characteristics $(n=222)$

\begin{tabular}{|c|c|c|c|}
\hline Variable & $n(\%)$ & $M(S D)$ & Range \\
\hline \multicolumn{4}{|l|}{ Health sector } \\
\hline Governmental & $123(55.4)$ & & \\
\hline University affiliated & $46(20.7)$ & & \\
\hline Private & $53(23.9)$ & & \\
\hline \multicolumn{4}{|l|}{ Inpatient/outpatient } \\
\hline Inpatient & $47(21.2)$ & & \\
\hline Outpatient & $175(78.8)$ & & \\
\hline \multicolumn{4}{|l|}{ Gender } \\
\hline Male & $124(55.9)$ & & \\
\hline Female & $98(44.1)$ & & \\
\hline \multicolumn{4}{|l|}{ Level of education } \\
\hline Less than high school & $46(20.7)$ & & \\
\hline High school & $54(24.3)$ & & \\
\hline Diploma & $86(38.7)$ & & \\
\hline Bachelor & $36(16.2)$ & & \\
\hline \multicolumn{4}{|l|}{ Marital status } \\
\hline Single & $10(4.5)$ & & \\
\hline Married & $170(76.6)$ & & \\
\hline Widow & $36(16.2)$ & & \\
\hline Separated & $6(2.7)$ & & \\
\hline \multicolumn{4}{|l|}{ Job } \\
\hline Full time & $67(30.2)$ & & \\
\hline Part time & $30(13.5)$ & & \\
\hline Unemployed & $103(46.4)$ & & \\
\hline Retired & $22(9.9)$ & & \\
\hline \multicolumn{4}{|l|}{ Family history of diabetes } \\
\hline Yes & $136(61.3)$ & & \\
\hline No & $86(38.7)$ & & \\
\hline \multicolumn{4}{|l|}{ Other co- morbidities } \\
\hline Yes & $112(50.5)$ & & \\
\hline No & $110(49.5)$ & & \\
\hline
\end{tabular}




\section{Diabetes complications}

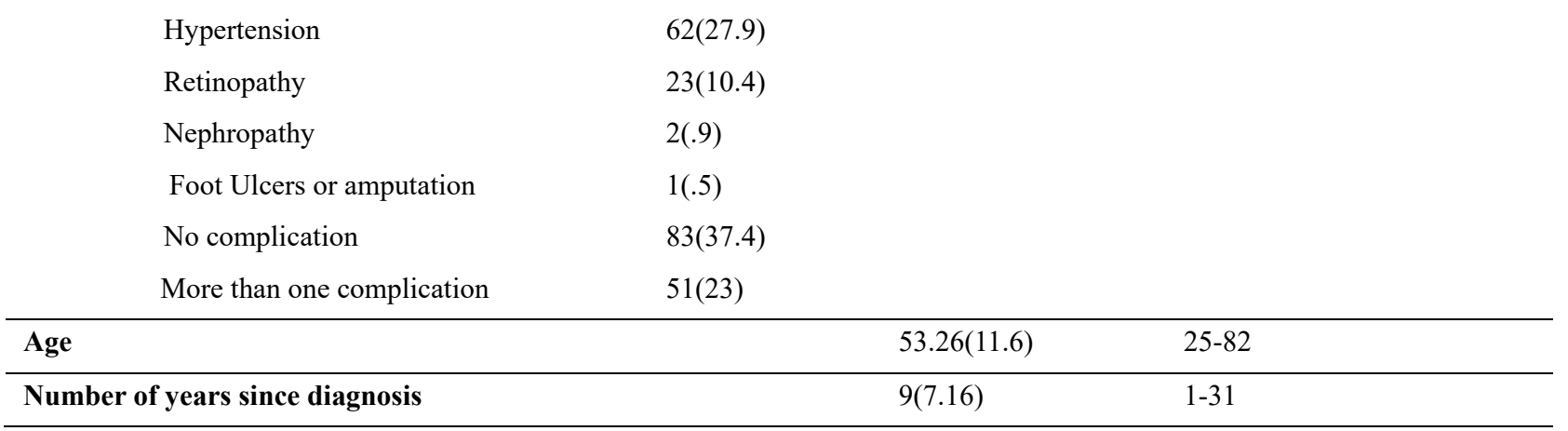

\section{2 Level of knowledge among individuals with T2DM}

Diabetes knowledge was measured using the diabetes knowledge test (DKT). Correct answers were marked 1 while incorrect answers were marked 0 . Out of 14 questions, the mean score for knowledge was 7.9(56.9\%) ranging from 1 to 14 (7.14\% to $100 \%)$. Knowledge scores were classified as follows: (1) high was any knowledge score above $74 \%$, (2) medium was any knowledge score $60-74 \%$ and (3) low was less than $60 \%$ (Kassahun et al., 2016). Among patients, $5.9 \%, 34.2 \%$ and $59.9 \%$ had high, medium and low level of knowledge, respectively. The overall knowledge score was $56.9 \%(\mathrm{SD}=13.1)$ which is considered low according to the previous classification (Kassahun et al., 2016) (Table 2).

Table 2. Knowledge among Patients Diagnosed with Type 2 Diabetes

\begin{tabular}{lll}
\hline Item No. & Item description & $\begin{array}{c}\text { Correct } \\
\text { answer\% }\end{array}$ \\
\hline 12 & Eating foods lower in fat decreases your risk for & $94.6 \% * *$ \\
13 & Numbness and tingling maybe symptoms of & $93.7 \%$ \\
14 & Which of the following is usually not associated with diabetes & $88.7 \%$ \\
6 & Which is the best method for testing blood glucose & $85.1 \%$ \\
11 & The best way to take care of your feet is to & $78.8 \%$ \\
9 & For a person in good control, what effect does exercise have on blood glucose? & $70.7 \%$ \\
5 & Glycosylated hemoglobin(hemoglobinA1)is a test that is a measure of your average blood & $62.6 \%$ \\
1 & glucose level for the past & $56.3 \%$ \\
2 & The diabetes diet is & 55.4 \\
3 & Which of the following is highest in carbohydrate? & $41 \%$ \\
10 & Which of the following is highest in fat & $29.7 \%$ \\
7 & Infection is likely to cause & $17.6 \%$ \\
\hline & Which should not be used to treat low blood glucose? & $16.2 \%$ \\
\hline
\end{tabular}

*lowest score.

** Highest score.

\subsection{Coping Strategies Employed by Patients Diagnosed with T2DM}

The coping strategies were assessed using diabetes coping measure (DCM) with a total of 21 items scored from 1 to 5 (agree strongly $=1$, agree a little $=2$, neutral $=3$, disagree a little $=4$, disagree strongly $=5$ ) with higher scores indicating greater use of the particular coping strategies by the patients. The 4 DCM subscale scores are converted from raw scores to a 0-100 range scores as follows: Subscale score $=(($ Mean subscale item score - minimum item 
score $) * 100) /($ maximum item score - minimum item score), where the minimum score for an item is 1 and the maximum is 5 . Therefore, subscale score $=(($ Mean subscale item score-1)*100) $/ 4$ (Welch et al., 1997).

Patients achieved higher scores in tackling spirit subscale with a mean 68.11 ( $\mathrm{SD}=17.15)$ while passive resignation had the lowest score with a mean of $38.63(\mathrm{SD}=21.35)$. Avoidance subscale showed a mean of $42.21(\mathrm{SD}=22.67)$ while Passive resignation subscale showed a mean score of $38.63(\mathrm{SD}=21.35)$. Finally, Diabetes integration subscale showed a mean score of $57.64(\mathrm{SD}=21.82)$ (Table 3).

Table 3. Coping strategies measured by DCM scale $(n=222)$

\begin{tabular}{|c|c|c|c|}
\hline Item No. & Item description & $M$ & $S D$ \\
\hline 1 & Most people would be a lot healthier if they followed a diabetic diet & $4.83 * *$ & .67 \\
\hline 4 & Because of my own experience, I can help educate other people about diabetes & 3.75 & 1.20 \\
\hline 9 & I believe that research will discover a cure for diabetes before long & 3.49 & 1.17 \\
\hline 12 & Clinical research is continually improving the treatments available for diabetes & 3.47 & 1.08 \\
\hline \multirow[t]{2}{*}{20} & My diabetes has caused me to think about life in a more positive way & $3.07 *$ & 1.34 \\
\hline & Tackling spirit & 3.72 & .68 \\
\hline 2 & $\begin{array}{l}\text { I am reluctant to visit my doctor for my regular diabetes checkup when I know I am in poor } \\
\text { blood glucose control }\end{array}$ & $2.07 *$ & 1.39 \\
\hline 3 & I dislike reading about diabetes because it only makes me worry more & 2.89 & 1.43 \\
\hline 5 & When my blood sugars are high I don't bother monitoring them as much & 2.61 & 1.33 \\
\hline 6 & It's difficult to fit regular blood glucose monitoring into my busy lifestyle & $3.08 * *$ & 1.32 \\
\hline \multirow[t]{2}{*}{8} & I am uncomfortable talking to people about my diabetes & 2.79 & 1.36 \\
\hline & Avoidance & 2.69 & .91 \\
\hline 7 & Whatever I do, diabetes complications will continue to ruin my health & 2.64 & 1.34 \\
\hline 10 & I feel like just giving in to my diabetes & 2.30 & 1.26 \\
\hline 11 & There is little I can do to control my blood glucose well & $3.14 * *$ & 1.27 \\
\hline 13 & Because of my illness, I cannot plan realistically for the future. & $2.15^{*}$ & 1.26 \\
\hline \multirow[t]{2}{*}{14} & I always seem to have poor blood sugars no matter what I do & 2.49 & 1.46 \\
\hline & Passive resignation & 2.54 & .85 \\
\hline 15 & Diabetes makes me feel different from everyone else & 3.13 & 1.31 \\
\hline 16 & I dislike being referred to as a "diabetic" & 3.20 & 1.39 \\
\hline 17 & Diabetes is the worst thing that has ever happened to me & 3.08 & 1.52 \\
\hline 18 & Most people would find it difficult to adjust to diabetes & $2.52 *$ & 1.30 \\
\hline 19 & Having diabetes over a long time changes your outlook on life for the worse & 3.22 & 1.44 \\
\hline \multirow[t]{2}{*}{21} & I think it is unfair that I should have diabetes when other people are so healthy & $4.67 * *$ & .94 \\
\hline & Diabetes integration & 3.30 & .87 \\
\hline
\end{tabular}

*lowest mean.

**highest mean.

\subsection{Relationship between Level of Knowledge and Coping Strategies}

Spearman test of correlation showed a statistically significant positive correlation between total knowledge score and tackling spirit coping $(r=.16, p<.05)$ which indicated that high knowledge score was associate with the use of tackling spirit coping strategies. Also, there was a statistically significant positive correlation between knowledge score and diabetes integration coping strategy, which indicates that high knowledge score was associated with the use of diabetes integration coping strategy $(r=.13, p<.05)$. A significant negative association was found between knowledge score and passive resignation coping strategy, which indicates that low knowledge 
score was associated with the use of passive resignation coping strategy $(r=-.17, p<.05)$. No significant correlation was found between knowledge score and avoidance coping strategy $(r=-.09, p>.05)$.

\section{Discussion}

\subsection{Level of Knowledge Among Individuals With T2DM}

This study assessed the level of knowledge among patients with type 2 diabetes. Findings in this study indicate that the level of knowledge among patient diagnosed with type 2 diabetes mellitus was low. Although this result cannot be generalized to all patients with diabetes in Jordan, it still gives a baseline for educational programs aiming to increase patient's knowledge regarding diabetes. Low level of knowledge in current study sample is consistent with other studies that assessed diabetes knowledge in different diabetes population around the world such as in Ethiopia (Kassahun et al., 2016) and India (Parimalakrishnan, Dussa, \& Sahay, 2015).

Several areas of knowledge deficits were identified in this study. Most of the questions regarding diabetes diet had the lowest scores. The majority (93\%) did not know what "free food" is, which is higher than the percentage (88\%) reported by Jasper et al. (2014), which indicate that the term "free food" is not understood in this culture. Approximately $84 \%$ of patients have incorrectly answered the question regarding unsweetened fruit juice (Question No. 7), this probably because of the misconception that any "unsweetened" food is the ideal food for patients with diabetes.

Although $56.3 \%$ knew that diabetes diet is the healthy diet for most people, $43.7 \%$ did not; this may related to the misconception that people with diabetes should only eat special diabetic food; while the fact is that healthy diet for people with diabetes is the same as other people with no diabetes (Akbar, Aqeel, Noman-Ul-Haq, \& Dhingra, 2016).

In this study, $62.3 \%$ of participants knew the relevance of $\mathrm{HbA1C}$ test, which is much higher than the percentage of $14.7 \%$ reported by Jasper et al (2014). Also, patient's knowledge of diabetes complications (questions 13 and 14) were relatively high. This could be attributed to fact that these symptoms were experience by these patients, or they have seen them in fellow patients (Al-Maskari et al., 2013).

\subsection{Coping Strategies Employed by Patients Diagnosed With T2DM}

The highest item mean score for passive resignation was $3.14(\mathrm{SD}=1.32)$ "There is little I can do to control my blood glucose well". This might reflect poor knowledge of diabetes self-management. Such patients need to be educated that diabetes is a controllable illness and patients can learn to live with it, manage it and avoid complications. However, the lowest item mean score was $2.15(\mathrm{SD}=1.26)$ "Because of my illness, I cannot plan realistically for the future", which indicate that patients do not let their illness affect their future plans.

Participant achieved higher scores in active coping (tackling spirit and diabetes integration coping), while passive coping (avoidance and passive resignation) had the lowest scores. These findings are consistent with the findings of other studies (i.e. Tuncay, Musabak, Gok, \& Kutlu, 2008). Karlsen, Oftedal and Bru (2012) found that $86 \%$ and $78 \%$ of patients reported occasionally or frequently using active coping and planning. A different results were concluded in a study on patients with Type 2 diabetes (Kokoszka, 2017) where patients used undifferentiated coping strategies, which means a combination of task-oriented, best solution-oriented, emotion-oriented and avoidance coping.

\subsection{Relationship Between Level of knowledge and Coping Strategies}

This study indicates a significant positive correlation between knowledge score and both tackling spirit and diabetes integration which reflects that the use of those coping strategies means better knowledge level among patients with Type 2 diabetes. While a significant negative correlation was found between knowledge score and passive resignation coping which indicates that this maladaptive coping is associated with lower level of knowledge among patients with Type 2 diabetes. Passive coping strategies has been associated with lower self-esteem, poorer glycemic control, and higher depressive symptoms and diabetes-specific distress (Luyckx, Seiffge-Krenke, \& Hampson, 2010).

\subsection{Limitation of the Study}

This study is the first descriptive study in Jordan that assessed the relationship between knowledge and coping strategies among patients diagnosed with type 2 diabetes mellitus. In addition, this study included patients from inpatient and outpatient clinics from different health sectors to enhance the generalizability of the findings. However, current study has some limitations that could affect the generalizability of the results, including: the study was limited to the central area of Jordan, south and north areas were not included. Despite the fact that the DKT questionnaire had been used in Jordan and other Arab countries, it is still considered a relatively difficult test. 
A development of a new, updated tool specific for our culture is recommended.

\section{Conclusion}

Knowledge among patients diagnosed with Type 2 diabetes is low. The employment of active coping strategies is associated with higher level of knowledge, while the employment of passive coping strategies is associated with lower level of knowledge. Efforts to improve patients' knowledge should be established with regards to appropriate coping strategies to be enforced and enriched through designing specific educational programs, along with continuous support and follow up.

\section{Implications}

\subsection{Implications for Clinical Practice}

This study reported low level of knowledge among patients with T2DM, and a significant relationship between knowledge and specific coping strategies. Current results have several implications for clinical practice. Nurses who provide care to patients with diabetes can play a crucial role in increasing the provision of updated diabetes education. Moreover, nurses are encouraged to perform assessment and support patients to reach out for a proper care and treatment and motivate them to take charge of their disease for their lifetime. Low level of knowledge identified in particular areas such as diet provides a clue to focus on diet-related information that patients required. Health care providers, as well, have to focus on improving the level of knowledge in this population through education and counseling. Self-monitoring and glucose control need to be enhanced among patients, while enforcing active coping strategies. Future educational programs should have a special consideration for patients' age, education level, and type of job. Also, coping strategies need to be integrated in education as the study findings have revealed a significant relationship between levels of knowledge and coping strategies. Finally, prevention of diabetes among healthy people and public can be achieved through health education and disease awareness campaigns.

\subsection{Implication for Nursing Curriculum and Research}

In order to improve nursing students' level of knowledge regarding diabetes, students should be adequately prepared to understand the aspect of diabetes education and how to perform appropriate assessment of patient's knowledge regarding diabetes and its care and provide proper education. Nursing Curricula should include the updated knowledge and skills to prepare students for their role in this aspect. Future interventional studies need be conducted to evaluate the effectiveness of the educational programs conducted to enhance knowledge regarding diabetes. Further studies need to be conducted to examine the role of coping strategies in dealing with chronic illness and the long term effect of those coping strategies on patients' well-being and quality of life.

\section{Funding}

This research didn't receive any specific grant from funding agencies in the public, commercial, or not-for-profit sectors.

\section{Acknowledgements}

The authors would like to thank all patients who participated in this study. In addition, thanks are extended to the organizations' leadership who granted access to participants in their organization.

\section{Competing Interests Statement}

The authors declare that they have no conflict of interest.

\section{References}

Ahola, A. J., \& Groop, P. H. (2013). Barriers to self-management of diabetes. Diabetic Medicine, 30(4), 413-420. https://doi.org/10.1111/dme.12105

Akbar, N., Aqeel, T., Noman-Ul-Haq, A. N., \& Dhingra, S. (2016). Assessment of Knowledge and Dietary Misconceptions among Diabetic Patients. Journal of Pharmacy Practice and Community Medicine, 2(1), 9-15. https://doi.org/10.5530/jppcm.2016.1.3

Al-Maskari, F., El-Sadig, M., Al-Kaabi, J. M., Afandi, B., Nagelkerke, N., \& Yeatts, K. B. (2013). Knowledge, attitude and practices of diabetic patients in the United Arab Emirates. PLoS One, 8(1), e52857. https://doi.org/10.1371/journal.pone.0052857

Faul, F., Erdfelder, E., Lang, A. G., \& Buchner, A. (2007). G*Power 3: a flexible statistical power analysis program for the social, behavioral, and biomedical sciences. Behavior Research Methods, 39(2), 175-191. https://doi.org/10.3758/BF03193146 
Fitzgerald, J. T., Funnell, M. M., Anderson, R. M., Nwankwo, R., Stansfield, R. B., \& Piatt, G. A. (2016). Validation of the Revised Brief Diabetes Knowledge Test (DKT2). The Diabetes Educator, 42(2), 178-187. https://doi.org/10.1177/0145721715624968

Gomes, M. B., Santos, D. C., Pizarro, M. H., Barros, B. S. V., de Melo, L. G. N., \& Negrato, C. A. (2018). Does knowledge on diabetes management influence glycemic control? A nationwide study in patients with type 1 diabetes in Brazil. Patient Preference and Adherence, 12, 53-62. https://doi.org/10.2147/PPA.S146268

International Diabetes Federation (IDF). (2015, October). Diabetes Atlas (7th ed). Retrieved from http://www.diabetesatlas.org/resources/2015-atlas.html

Islam, S. M., Niessen, L. W., Seissler, J., Ferrari, U., Biswas, T., Islam, A., \& Lechner, A. (2015). Diabetes knowledge and glycemic control among patients with type 2 diabetes in Bangladesh. Springerplus, 4, 284. https://doi.org/10.1186/s40064-015-1103-7

Jasper, U. S., Ogundunmade, B. G., Opara, M. C., Akinrolie, O., Pyiki, E. B., \& Umar, A. (2014). Determinants of diabetes knowledge in a cohort of Nigerian diabetics. Journal of Diabetes \& Metabolic Disorders, 13(1), 39. https://doi.org/10.1186/2251-6581-13-39

Karlsen, B., Oftedal, B., \& Bru, E. (2012). The relationship between clinical indicators, coping styles, perceived support and diabetes-related distress among adults with type 2 diabetes. Journal of advanced nursing, 68(2), 391-401. https://doi.org/10.1111/j.1365-2648.2011.05751.x

Kassahun, T., Gesesew, H., Mwanri, L., \& Eshetie, T. (2016). Diabetes related knowledge, self-care behaviours and adherence to medications among diabetic patients in Southwest Ethiopia: a cross-sectional survey. BMC Endocrine Disorders, 16(1), 28. https://doi.org/10.1186/s12902-016-0114-x

Kokoszka, A. (2017). Treatment adherence in patients with type 2 diabetes mellitus correlates with different coping styles, low perception of self-influence on disease, and depressive symptoms. Patient Preference and Adherence, 11, 587-595. https://doi.org/10.2147/PPA.S124605

Luyckx, K., Seiffge-Krenke, I., \& Hampson, S. E. (2010). Glycemic control, coping, and internalizing and externalizing symptoms in adolescents with type 1 diabetes: a cross-lagged longitudinal approach. Diabetes Care, 33(7), 1424-1429. https://doi.org/10.2337/dc09-2017

Ministry of Healh (MOH, April). (2015). Retrieved from http://apps.moh.gov.jo/reports/headermain.jsp?print_parameter=yes\&lang_parameter=arabic

Mulligan, K., McBain, H., Lamontagne-Godwin, F., Chapman, J., Haddad, M., Jones, J., . . Simpson, A. (2017). Barriers and enablers of type 2 diabetes self-management in people with severe mental illness. Health Expectations, 20(5), 1020-1030. https://doi.org/10.1111/hex.12543

Parimalakrishnan, S., Dussa, K., \& Sahay, R. (2015). Assessment of diabetes knowledge using diabetes knowledge questionnaire among people with type 2 diabetes mellitus. Asian Journal of Pharmaceutical and Clinical Research, 8(2), 254-256.

Ridosh, M. M., Roux, G., Meehan, M., \& Penckofer, S. (2017). Barriers to Self-Management in Depressed Women With Type 2 Diabetes. The Canadian Journal of Nursing Research, 49(4), 160-169. https://doi.org/10.1177/0844562117736699

Tuncay, T., Musabak, I., Gok, D. E., \& Kutlu, M. (2008). The relationship between anxiety, coping strategies and characteristics of patients with diabetes. Health and Quality of Life Outcomes, 6, 79-79. https://doi.org/10.1186/1477-7525-6-79

Unnikrishnan, R., \& Mohan, V. (2016). Diabetes in the tropics: prevalent, increasing and a major public health problem. Transactions of the Royal Society of Tropical Medicine and Hygiene, 110(5), 263-264. https://doi.org/10.1093/trstmh/trw021

Welch, G. W. (1994). The Diabetes Coping Measure: A measure of cognitive and behavioural coping specific to diabetes. In C. Bradley (Ed.), Handbook of psychology and diabetes: A guide to psychological measurement in diabetes research and practice (pp. 391-404). London: Harwood Academic Publishers.

Welch, G. W., Jacobson, A. M., \& Polonsky, W. H. (1997). The Problem Areas in Diabetes Scale. An evaluation of its clinical utility. Diabetes Care, 20(5), 760-766. https://doi.org/10.2337/diacare.20.5.760

World Health Organization (WHO). (n.d.). Process of Translation and Adaptation of Instruments. Retrieved November 12, 2016, from http://www.who.int/substance_abuse/research_tools/translation/en/ 
Zhu, M., Li, J., Li, Z., Luo, W., Dai, D., Weaver, S. R., . . Fu, H. (2015). Mortality rates and the causes of death related to diabetes mellitus in Shanghai Songjiang District: an 11-year retrospective analysis of death certificates. BMC Endocrine Disorders, 15(1), 45. https://doi.org/10.1186/s12902-015-0042-1

\section{Copyrights}

Copyright for this article is retained by the author(s), with first publication rights granted to the journal.

This is an open-access article distributed under the terms and conditions of the Creative Commons Attribution license (http://creativecommons.org/licenses/by/4.0/). 